\title{
Proteinosis alveolar
}

\author{
Alveolar proteinosis
}

\author{
Carlos Bula G., MD. ${ }^{(1)}$; Ernesto Santiago H., MD. ${ }^{(2)}$; Brenda Martínez O., MD. ${ }^{(3)}$; \\ MELISA REYES, MD. ${ }^{(4)}$; RICARDO PABÓN R., MD. ${ }^{(5)}$
}

\section{Resumen}

La proteinosis alveolar pulmonar es una enfermedad rara, caracterizada por la acumulación de surfactante en el interior del espacio alveolar, que conduce a una alteración del intercambio gaseoso. El curso clínico es variable, abarcando desde la resolución espontánea hasta la falla respiratoria y la muerte. Se han descrito tres formas: la genética, la secundaria y la primaria o idiopática; esta última es la más frecuente en la población adulta. La fisiopatología está dada por un proceso autoinmune dirigido contra el factor estimulante de colonias de granulocitos y macrófagos (GM-CSF), que condiciona un defecto funcional en los macrófagos que conlleva acumulación intraalveolar de surfactante y manifestaciones infecciosas pulmonares y extrapulmonares, a menudo por gérmenes no usuales.

El conjunto de datos aportados por las imágenes, la disociación clínico-radiológica y material de lavado broncoalveolar, en general son suficientes para el diagnóstico, aunque en ocasiones se requiere de la histopatología para su confirmación. El tratamiento recomendado es el lavado pulmonar total. La mortalidad es baja y se relaciona fundamentalmente con las complicaciones infecciosas. Se describe caso clínico de proteinosis alveolar pulmonar, cuyo diagnóstico requirió estudio histopatológico de biopsia pulmonar por toracoscopia y se hace una revisión de la literatura.

Palabras clave: proteinosis alveolar, disnea, lavado pulmonar total.

\section{Abstract}

Pulmonary alveolar proteinosis is a rare disease characterized by accumulation of surfactant within the alveolar space, which leads to a deranged gas exchange. Its clinical course is variable, ranging from spontaneous resolution to respiratory failure and death. Three forms have been described: genetic, secondary, and primary or idiopathic. The latter is the most common form in the adult population. The pathophysiology is given by an autoimmune process against the granulocyte-macrophage colony-stimulating factor (GM-CSF); this process conditions a functional impairment of macrophages that leads to accumulation of surfactant within the alveoli, as well as pulmonary and extrapulmonary infectious manifestations, often due to unusual germs.

The bulk of data yielded by image studies, clinical-radiological dissociation, and bronchoalveolar lavage material is usually enough for reaching diagnosis, although histopathology is sometimes required for confirmation. Recommended treatment is total pulmonary lavage. The mortality is low and is fundamentally related to infectious complications. We describe a case of pulmonary alveolar proteinosis that required confirmation by histopathologic study of thoracoscopic lung biopsy. We also offer a review of the literature.

Keywords: alveolar proteinosis, dyspnea, total pulmonary lavage.

\footnotetext{
${ }^{(1)}$ Médico Neumólogo. Coordinador Departamento de Neumología y Docente de Postgrado Hospital Fundación Universidad Metropolitano (FHUM). Barranquilla, Colombia.

${ }^{(2)}$ Médico Internista Neumólogo: Clínica General del Norte. Barranquilla, Colombia. ${ }^{(3)}$ Médico Internista: Fundación Hospital Universidad Metropolitana de Barranqui1la. Barranquilla, Colombia.

${ }^{(4)}$ Residente de tercer año de Medicina Interna. Universidad Libre de Barranquilla. Barranquilla, Colombia.

${ }^{(5)}$ Residente de segundo año de Medicina Interna FHUM. Fundación Universitaria Hospital Metropolitano, Barranquilla, Colombia.

Correspondencia: Carlos Bula G., correo electrónico: cjbulag@hotmail.com Recibido: 12/06/16. Aceptado: 15/11/16.
} 


\section{Introducción}

La proteinosis alveolar pulmonar es una enfermedad rara, que se caracteriza por la acumulación alveolo-pulmonar de un material lipoproteico derivado del surfactante alveolar, lo que provoca el deterioro consiguiente del intercambio gaseoso.

Fue descrita originalmente por los patólogos Rosen, Castleman y Liebbow, entre 1953 y 1958 basándose en el estudio tisular de 27 pacientes identificados, y desde ese momento se han reportado en la literatura menos de 1.000 casos $(1,2)$.

Su prevalencia en su forma adquirida, que representa el $90 \%$ de los casos, es de 0,37 por 100.000 personas y en estudios recientes la incidencia estimada es de 1:2.000.000 de casos nuevos/año. Es más frecuente en hombres, con una relación $\mathrm{H} / \mathrm{M}$ $(2,6: 1)$ y de preferencia en la tercera y cuarta décadas de la vida, y en población fumadora en el $70 \%$ de los casos (2).

Rosen, Castleman y Liebbow describieron la apariencia histológica de la proteinosis alveolar pulmonar caracterizada por la acumulación de abundante material eosinofílico que consistía en lípidos, proteínas y carbohidratos (3).

La proteinosis alveolar pulmonar se puede clasificar en tres categorías, dependiendo de la etiología: autoinmune (previamente llamada idiopática o primaria), responsable del $88 \%$ de los casos, secundaria, del $10 \%$ y genética del $2 \%$ restante (4-6).

\section{Congénita}

Se han descrito hasta tres mutaciones diferentes que pudiesen explicar la proteinosis alveolar pulmonar en neonatos (proteína B del surfactante, GMCSF y cadena $\beta$ del receptor de GM) $(7,8)$; pero, en la mayoría, la base genética no queda definida. Sin trasplante de pulmón, estos pacientes mueren entre los 3 y 6 meses de edad.

\section{Secundaria}

Ocurre en sujetos que presentan condiciones asociadas con deterioro de la función de los macrófagos como neoplasias (9) (especialmente de origen hematológico como leucemias y linfomas), tratamientos inmunosupresores o citotóxicos (10), infección por el virus de la inmunodeficiencia humana (VIH) (11), nocardia, criptococco, mucormicosis, histoplasmosis, micobacterias, Pneumocystis jirovecii y por la inhalación de partículas; por ejemplo, se ha reportado un aumento de la frecuencia de proteinosis alveolar pulmonar luego de importantes exposiciones a polvo de sílice en arenadores, así como por inhalación de aluminio y titanio (12).

\section{Adquirida}

También llamada primaria o idiopática, es la más frecuente, constituyendo casi el $90 \%$ de los casos reportados. Está caracterizada y determinada por la presencia de anticuerpos anti GM-CSF que contribuirían a la acumulación del surfactante pulmonar por la existencia de macrófagos no funcionales.

Debido a la disfunción de los macrófagos, los pacientes con proteinosis alveolar pulmonar presentan riesgo de desarrollar infecciones secundarias a diversos microorganismos inusuales e infecciones oportunistas como: nocardia, micobacterias y aspergillus.

\section{Etiología y patogénesis}

La homeostasis del surfactante es mantenida por el balance entre la producción y el aclaramiento. La depuración por los macrófagos alveolares requiere de la estimulación del GM-CSF (13). Los macrófagos alveolares son células claves en la depuración del surfactante pulmonar; sin embargo, ésta no es su única función.

Estos poseen una ubicación privilegiada y están dotados de un amplio repertorio de receptores de reconocimiento de patrones moleculares asociados a patógenos (PAMPs) que le permiten censar la presencia de microorganismos y activar la producción de citoquinas y quimiocinas específicas para iniciar la respuesta inflamatoria.

La clave en la iniciación y el desarrollo de la proteinosis alveolar pulmonar está dada por la obstaculización en la señalización del GM-CSF y su receptor. Este hallazgo fue demostrado en modelos de ratón 
con deficiencias en la expresión del GM-CSF o de la cadena $\beta$ de su receptor. El déficit de GM-CSF o la falta de la señalización de su receptor, no sólo afecta la depuración del surfactante sino también la expresión de receptores de PAMPs, adhesión celular y la producción de sustancias microbicidas y citoquinas. De este modo, los pacientes con proteinosis alveolar pulmonar pueden ser más susceptibles a infecciones por estreptococos y Pneumocystis jirovecii (15).

En 1999 se describieron autoanticuerpos neutralizantes contra el GM-CSF tanto en el suero como en el lavado broncoalveolar (LBA) de pacientes con proteinosis alveolar pulmonar (14). Los procesos generados a partir de la unión del GM-CSF con su receptor celular, se verifican a través de un factor de transcripción llamado PU-1, que modula la expresión de numerosos genes, decisivos en la diferenciación final de los macrófagos alveolares.

Estos anticuerpos reconocen con alta afinidad al GM-CSF; lo secuestran, dejando sin ligando a su receptor, por lo tanto la inhibición del acoplamiento del GM-CSF a su receptor por la acción de los anticuerpos neutralizantes, induce un estado de déficit funcional del macrófago alveolar para la remoción del surfactante y la actividad antibacteriana (16).

\section{Cuadro clínico y exámenes complementarios}

Los síntomas, de presentación lenta y progresiva, son fundamentalmente disnea $(64 \%)$ y tos (41\%) (que puede o no ser productiva), los cuales pueden además por lo inespecíficos, estar presentes durante meses o hasta años sin generar estudios que sugieran el diagnóstico. Ocasionalmente, presentan febrícula, pérdida de peso y raramente hemoptisis (en estos casos se debe descartar infección pulmonar). Aproximadamente un tercio de los pacientes son asintomáticos (17).

Es característica la disociación entre la escasez de síntomas y hallazgos físicos con las manifestaciones radiológicas, de compromiso extenso y bilateral. Los signos clínicos más frecuentes son los crépitos (entre 28 y $50 \%$ de los pacientes) y la cianosis (entre 14 y 25\%). El menos común es el hipocratismo digital.

\section{Diagnóstico}

Se debe sospechar proteinosis alveolar pulmonar en todo paciente con disnea crónica, progresiva, con una radiografía de tórax que evidencie opacidades alveolares bilaterales y simétricas con acentuación perihiliar similar a un edema de pulmón cardiogénico, sin cardiomegalia ni derrame pleural. En la tomografía computada de alta resolución (TCAR) se observa típicamente atenuación en vidrio esmerilado asociada con engrosamiento de los septos inter e intralobulillares conformando el patrón típico de "crazy paving" o en empedrado. El patrón en "crazy paving" no es específico para proteinosis alveolar pulmonar ya que puede estar asociado a otras entidades como distrés respiratorio, hemorragia alveolar difusa, edema pulmonar cardiogénico, toxicidad pulmonar por fármacos y neumonía lipoidea.

El diagnóstico por lo general se realiza mediante LBA sin necesidad de biopsia en la mayoría de los casos. Por tanto, éste se basa en la combinación del cuadro clínico, la TCAR y las características del LBA. En algunas ocasiones, se incluye además la biopsia transbronquial y finalmente la dosificación en suero o LBA de anticuerpos anti GM-CSF mediante ELISA o aglutinación de látex. La sensibilidad y especificidad para aglutinación de látex en el suero es de $100 \%$ y $98 \%$ respectivamente $(18,19)$.

La espirometría muestra un defecto ventilatorio restrictivo de severidad variable.

Los gases arteriales revelan hipoxemia de moderada a severa. Los hallazgos de laboratorio son inespecíficos. Títulos elevados de anticuerpos neutralizantes del GM-CSF, tanto en el plasma como en el LBA, resultan útiles para el diagnóstico, pronóstico y seguimiento (20).

El LBA tiene uno de los más altos rendimientos diagnósticos en la proteinosis alveolar pulmonar. El fluido recuperado luego de la instilación de solución fisiológica es extremadamente turbio o lechoso y contiene un material denso y blanquecino que sedimenta con bastante rapidez cuando se deja en reposo. La tinción del mismo demuestra que es paucicelular con un material de fondo granular basófilo amorfo, PAS positivo (21). Presenta macrófagos 
espumosos con abundantes inclusiones intracelulares y el análisis ultraestructural revela numerosos cuerpos lamelares.

El tratamiento estándar de esta patología es el lavado pulmonar total (LPT) (22). Este fue introducido por Ramírez en 1965 y se realiza por medio de un tubo endotraqueal de doble lumen que permite el aislamiento del pulmón lavado del contralateral que ventilará al paciente durante el procedimiento, irrigándolo con cristaloides tibios, requiriéndose en promedio entre 10 a $15 \mathrm{~L}$ para lograr el aclaramiento del líquido extraído.

El LPT se indica tras confirmación histológica y si cumple alguno de los siguientes criterios (23): $\mathrm{PaO} 2$ en reposo al nivel del mar $<65 \mathrm{~mm} \mathrm{Hg}(54,10$ $\mathrm{mm} \mathrm{Hg}$ ); gradiente alvéolo-arterial de $\mathrm{O} 2>40 \mathrm{~mm}$ $\mathrm{Hg}$ (43 mm Hg); fracción de shunt medio> 10-12\%; disnea severa e hipoxemia en reposo o en ejercicio. La mayoría evoluciona de manera favorable con un único LPT, pero en una minoría de casos, se necesitan más procedimientos.

Como todo procedimiento clínico, no está exento de complicaciones, entre las cuales se encuentran neumonía, sepsis, hidroneumotórax y síndrome de dificultad respiratoria del adulto (24). Globalmente, el LPT es una opción terapéutica eficaz en aproximadamente dos tercios de los casos.

Luego del LPT, en la mayoría de casos, se observa una mejoría rápida (en la primera semana) en la $\mathrm{PaO} 2$, el gradiente alvéolo-arterial de oxígeno y la CVF. Otros parámetros como la DLCO y la distancia recorrida en la prueba de marcha de los $6 \mathrm{mi}-$ nutos, mejoran con el correr de los meses con una meseta a los 6-12 meses (27).

Tras el lavado pulmonar total, muchos grupos plantean la opción del tratamiento inhalado con GM-CSF a dosis de 250 microgramos dos veces por día, cada 15 días durante 12 semanas o vía subcutánea, pero se prefiere la vía inhalatoria porque facilita la llegada del fármaco al sitio de acción $(5,13,15$, 17, 26, 27). A pesar de que en estudios recientes la proporción de pacientes que responden a esta terapia es inferior a la del lavado pulmonar completo (23), se recomienda su uso como tratamiento complementario al LPT, especialmente en caso de respuesta insuficiente a este último.

Si fracasan tanto el lavado pulmonar total como la terapia combinada, se han descrito ensayos clínicos fase 2 en pacientes con proteinosis alveolar pulmonar autoinmune que demuestran mejoría con el tratamiento intravenoso con rituximab, anticuerpo monoclonal contra el antígeno CD20 de los linfocitos $\mathrm{B}$ útil en diversas enfermedades de etiología autoinmune (25). Dicho efecto se conseguiría con el descenso de los niveles de los mencionados linfocitos B, así como de los anticuerpos anti GM-CSF IgG en el pulmón afectado, disminuyendo por tanto el cúmulo del surfactante pulmonar al que contribuirían.

La plasmaféresis podría ser de ayuda en pacientes no respondedores una vez agotados los demás recursos (28).

En el caso de la proteinosis alveolar pulmonar secundaria, su tratamiento y pronóstico estarán determinados por la evolución de la enfermedad subyacente.

\section{Caso clínico}

Paciente de sexo femenino, de 24 años de edad, con escolaridad bachillerato completo y ocupación actual ama de casa, sin antecedentes patológicos conocidos o referidos de importancia y antecedentes familiares de diabetes mellitus 2 (abuela materna), quien consultó por cuadro clínico de varios meses de evolución caracterizado por tos seca persistente, y aparición posterior de disnea de grandes a medianos esfuerzos que la motivó a asistir en varias oportunidades al servicio de medicina general y a urgencias, donde recibió tratamiento con antihistamínicos orales, salbutamol y beclometasona inhalados. Persistió con la sintomatología, presentando disnea de pequeños esfuerzos, por lo que consultó al servicio de urgencias, donde fue valorada por Médico internista, quien al examen físico la halló desaturada y con estertores finos bilaterales. Fue hospitalizada y recibió oxígeno por cánula nasal; adicionalmente se le ordenaron estudios. 


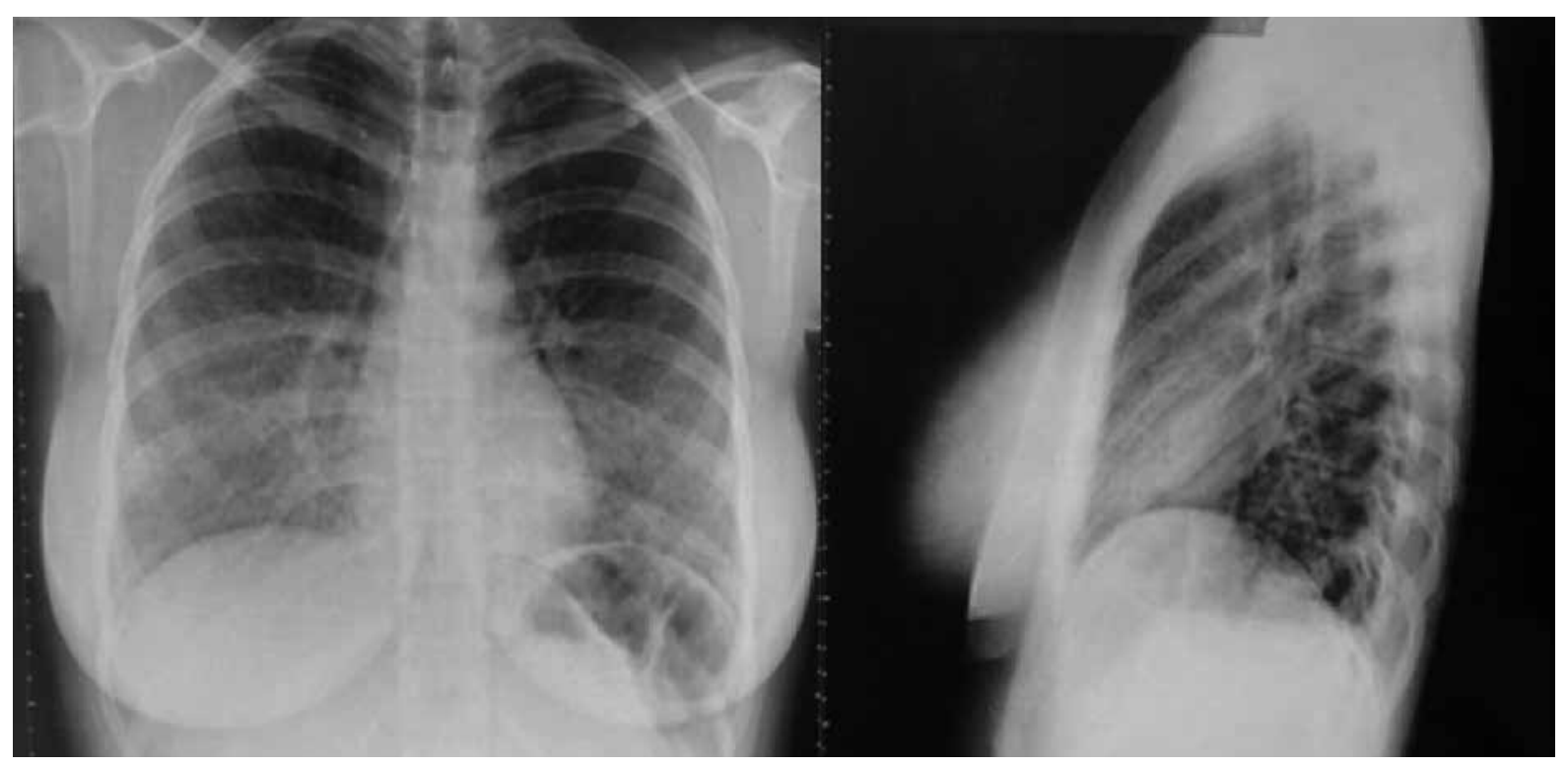

Figura 1. Inflitrados parahiliares y basales bilaterales.

La radiografía de tórax mostró infiltrado intersticial en vidrio esmerilado, parahiliar y basal bilateral (figura 1).

La tomografía computarizada de tórax evidenció cambios por patología pulmonar intersticial bilateral difusa.

El hemograma no mostró anemia ni alteración de las otras líneas celulares; no se reportaron alteraciones hidroelectrolíticas, glicemia normal

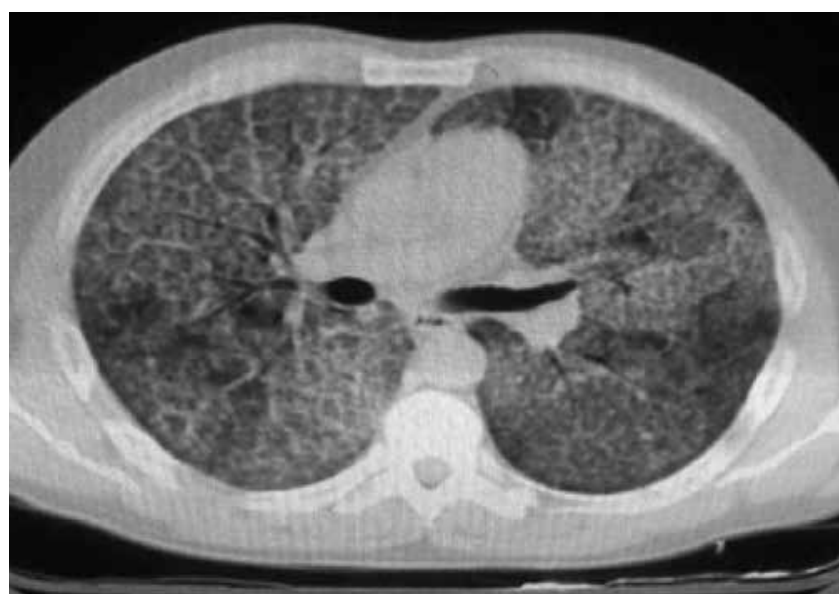

Fígura 2. Lesiones descritas como "crazy paving". y función renal conservada, perfil inmunológico (ANAS, antiDNA doble cadena, ENAS, ANCAS, C3-C, antihistonas) dentro de parámetros normales, además de VIH y baciloscopia negativos.

La espirometría mostró patrón restrictivo severo con respuesta al broncodilatador.

La TACAR y la radiografía de tórax (figuras 2 y 3) evidencian densidades en vidrio esmerilado bien delimitadas generando un aspecto "geográfico",

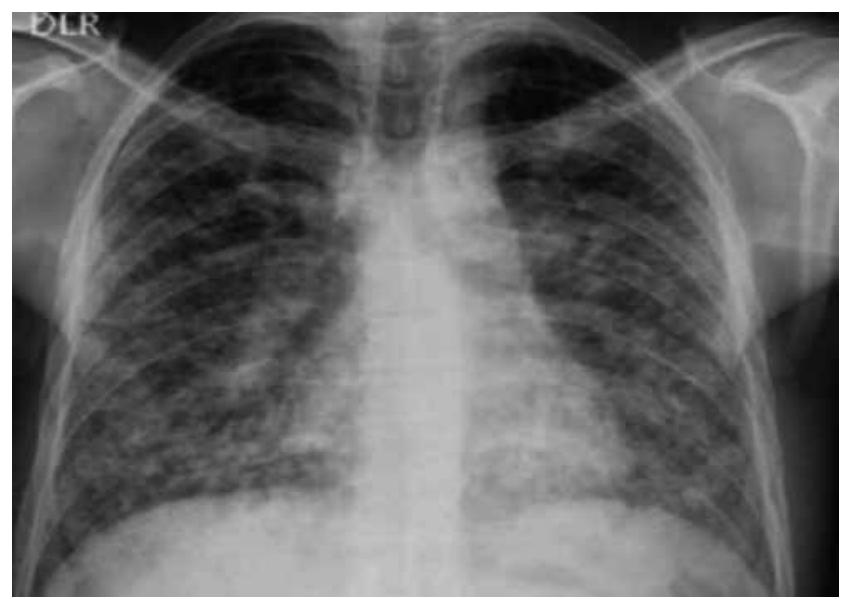

Figura 3 . Progresión de los inflitrados difusos bilaterales. 
con engrosamiento de los septos inter e intralobulillares, patrón en "crazy paving" y broncograma aéreo rodeado de vidrio esmerilado.

En la fibrobroncoscopia, el cepillado, lavado y biopsia bronquial mostraron escasas células epiteliales cilíndricas, sin cambios morfológicos específicos. Por lo anterior se realizó biopsia pulmonar por videotoracoscopia (figura 4) que reportó: pulmón con pérdida de su arquitectura normal, ocupación difusa de la luz de los alveólos por material proteináceo PAS positivo, cuerpos eosinofílicos, bronquiolos material proteináceo en la luz, no hay evidencia de granulomas ni tumor; las coloraciones de ZN y Grocott fueron negativas para BAAR y hongos, confirmándose el diagnóstico de proteinosis alveolar pulmonar.

Presentó deterioro de su cuadro clínico y funcional e ingresó a la unidad de cuidado intensivo, donde fue programada para LPT (figuras 5 y 6), el cual toleró sin complicaciones. Cuarenta y ocho horas después fue extubada y trasladada a piso, donde evolucionó de manera satisfactoria. Posteriormente, se le dio de alta con indicación de oxígeno domiciliario y seguimiento por consulta externa de Neumología.

Continúa en seguimiento por Neumología con evolución clínica, radiológica y funcional favorables.

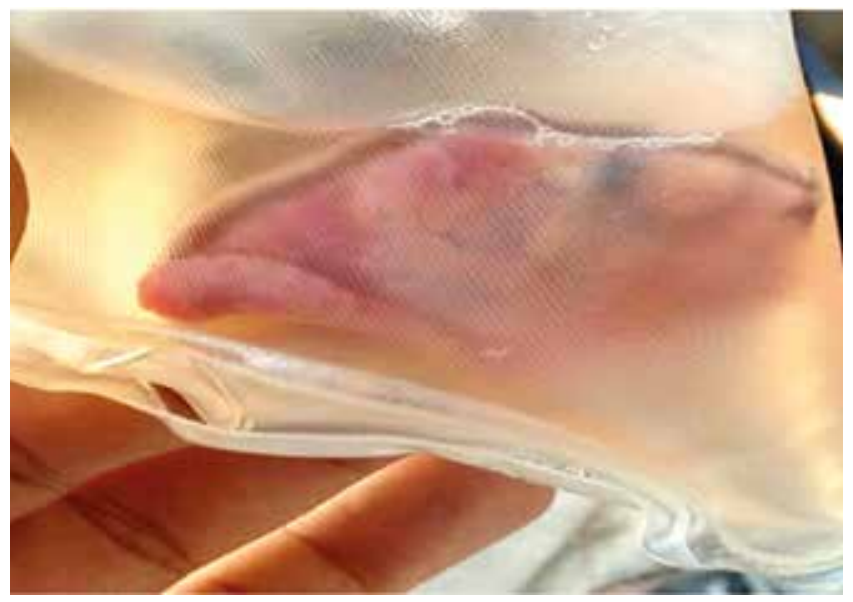

Figura 4. Cuña de pulmón que mide $4 \times 3 \mathrm{~cm}$. Al corte muestra aspecto de consolidación difusa.
La TACAR realizada un año después del LAP total mostró resolución casi total del infiltrado intersticial en vidrio esmerilado (figura 7); igualmente, la espirometría mostró normalización de los parámetros y la DCLO se halló moderadamente disminuida pero con mejoría respecto a las iniciales.

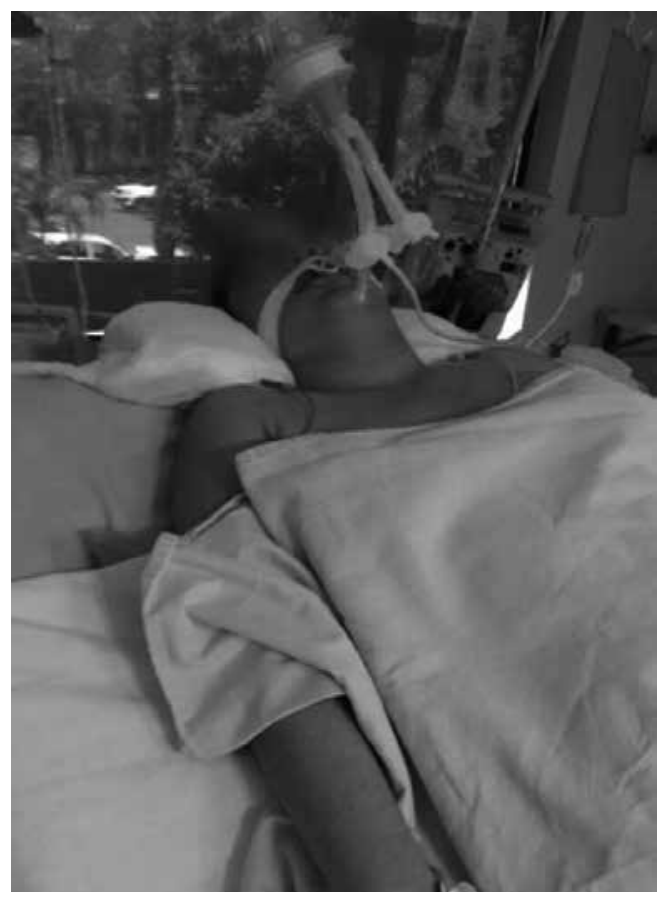

Figura 5. Lavado pulmonar total. Se observa tubo endotraqueal de doble lumen. Publicado con autorización del paciente.

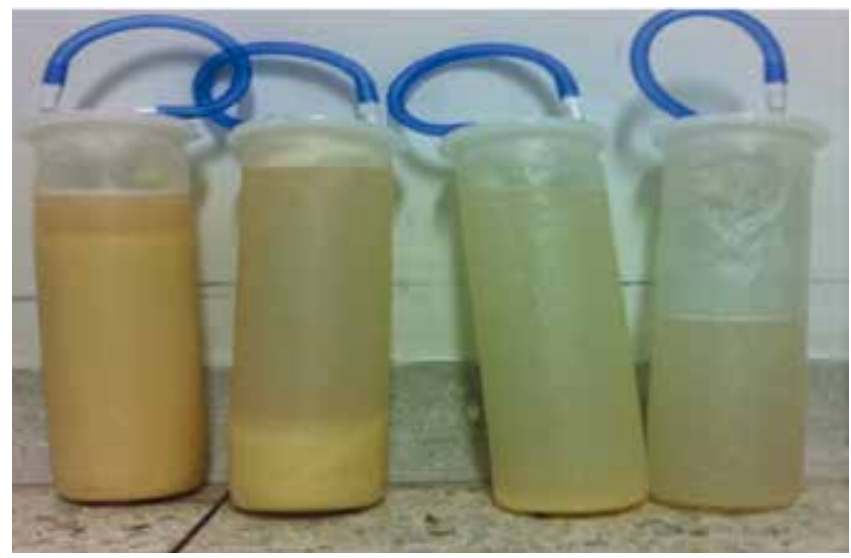

Figura 6. Lavado pulmonar total. A medida que va progresando el lavado, el material recuperado se aclara de manera paulatina. 


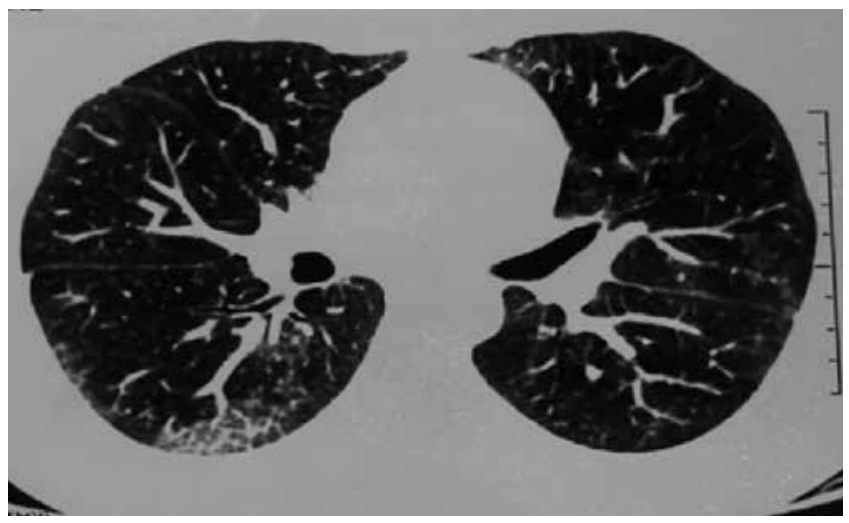

Figura 7. TACAR tomada un año después de los procedimientos, donde se aprecia resolución casi completa del infiltrado intersticial en vidrio esmerilado.

\section{Conclusiones}

Se presenta un caso de proteinosis alveolar primaria, probablemente autoinmune, en el que la clínica y los estudios radiológicos, mostraron el deterioro progresivo de la enfermedad con alto riesgo de falla respiratoria que ameritó el manejo en unidad de cuidados intensivos y la aplicación del tratamiento estándar de Lavado pulmonar total que mostró una notable mejoría clínica, radiológica, funcional y en la calidad de vida de la paciente. En la actualidad ha evolucionado satisfactoriamente sin signos de sintomatología respiratoria.

\section{Bibliografía}

1. Rosen SH, Castleman B, Liebbow AA. Pulmonary alveolar proteinosis. N Engl J Med. 1958; 258:1124-42.

2. Seymour JF, Presneill JJ. Pulmonary alveolar proteinosis: progress in the first 44 years. Am J Respir Crit Care Med. 2002; 166:215-35.

3. Larson RK, Gordinier R. Pulmonary alveolar proteinosis: report of six cases, review of the literature, and formulation of a new theory. Ann Intern Med. 1965;62:292-312.

4. Kumar V, Abbas AK, Fausto N, Aster JC. Robbins y Cotran. Patología estructural y funcional, $7^{\mathrm{a}}$ ed. Ed. Elsevier; 2007. p. 745-6.

5. Borie R, Danel C, Debray MP, Taille C, Dombret MC, Aubier M, et al. Pulmonary alveolar proteinosis. Eur Respir Rev. 2011; 20:98-107.

6. Inoue Y, Trapnell BC, Tazawa R, Arai T, Takada T, Hizawa N. Characteristics of a large cohort of patients with autoimmu- ne pulmonary alveolar proteinosis in Japan. Am J Respir Crit Care Med. 2008;177: 752-62.

7. Teja K, Cooper PH, Squires JE, Schnatterly PT. Pulmonary alveolar proteinosis in four siblings. $\mathrm{N}$ Engl $\mathrm{J}$ Med. 1981;305(23): 1390-2.

8. Suzuki T, Sakagami T, Young LR, Carey BC, Wood RE, Luisetti $\mathrm{M}$, et al. Hereditary pulmonary alveolar proteinosis: pathogenesis, presentation, diagnosis, and therapy. Am J Respir Crit Care Med. 2010; 182(10): 1292-304.

9. Schiller V, Aberle DR, Aberle AM. Pulmonary alveolar proteinosis. Occurrence with metastatic melanoma to lung. Chest. 1989; 95(2): 466-7.

10. Bedrossian CW, Luna MA, Conklin RH, Miller WC. Alveolar proteinosis as a consequence of immunosuppression. A hypothesis based on clinical and pathologic observations. Hum Pathol. 1980;11(5 Suppl):527-35.

11. Ruben FL, Talamo TS. Secondary pulmonary alveolar proteinosis occurring in two patients with acquired immune deficiency syndrome. Am J Med. 1986;80(6):1187-90.

12. Keller CA, Frost A, Cagle PT, Abraham JL. Pulmonary alveolar proteinosis in a painter with elevated pulmonary concentrations of titanium. Chest. 1995;108(1):277-80.

13. Chan ED, King TE. Diagnosis and treatment of pulmonary alveolar proteinosis in adults. Uptodate. [Sede web]. Jun, 2013. Waltham, Massachusetts. com/

14. Kitamura T, Tanaka N, Watanabe J, et al. Idiopathic pulmonary alveolar proteinosis as an autoimmune disease with neutralizing antibody against granulocyte/macrophage colonystimulating factor. J Exp Med. 1999;190:875-80.

15. Paine R, Preston AM, Wilcoxen S, Jin H, Siu BB, Morris SB, et al. Granulocyte-macrophage colony-stimulating factor in the innate immune response to Pneumocystis carinii pneumonia in mice. J Immunol. 2000; 164(5): 2602-9.

16. Kumar V, Abbas AK, Fausto N, Aster JC. Robbins y Cotran. Patología estructural y funcional, $7^{\mathrm{a}}$ ed. Ed. Elsevier; 2007. p. 745-6.

17. Rodríguez J, Rodríguez E, Sánchez A. Proteinosis alveoloar. Respuesta al tratamiento con factor estimulante de colonias de granulocitos y macrófagos por vía inhalada. Arch Bronconeumol. 2009;45(3):150-2.

18. Bonella F, Bauer PC, Griese M, Ohshimo S, Guzman J, Costabel U. Pulmonary alveolar proteinosis: new insights from a single-center cohort of 70 patients. Respir Med. 2011;105(12):1908-16.

19. Leth S, Bendstrup E, Vestergaard H, Hilberg O. Autoimmune pulmonary alveolar proteinosis: treatment options in year 2013. Respirology. 2013;18(1):82.

20. Lin FC, Chang GD, Chern MS, Chen YC,Chang SC. Clinical significance of anti-GMCSF antibodies in idiopathic pulmonary alveolar proteinosis. Thorax. 2006;61:528-34.

21. Trapnell BC, Uchida K. Pulmonary alveolar proteinosis. Eur Respir Mon. 2009;46(12):208-24. 
22. Huizar I, Kavuru M. Alveolar proteinosis syndrome: pathogenesis, diagnosis and management. Curr Opin Pulm Med. 2009;15(5):491-8.

23. Chan ED, King TE. Diagnosis and treatment of pulmonary alveolar proteinosis in adults. Uptodate. [Sede web]. Jun, 2013. Waltham, Massachusetts.

24. Vancheeswaran R, Morgan C, Wells A. Pulmonary alveolar proteinosis. Investigation and management $-\mathrm{A}$ review. Clin Pulmonary Med. 2007;14(6):360-4.

25. Kavuru MS, Malur A, Marshall I, Barna BP, Meziane M, Huizar I, et al. An open-label trial of rituximab therapy in pulmonary alveolar proteinosis. Eur Respir J. 2011;38:1361-7.

26. Tazawa R, Trapnell BC, Inoue Y, Arai T, Takada T, Nasuhara $\mathrm{Y}$, et al. Inhaled granulocyte/macrophage-colony stimulating factor as therapy for pulmonary alveolar proteinosis. Am J Respir Crit Care Med. 2015;181(12):1345-54.

27. Beccaria M, Luisetti M, Rodi G, Corsico A, Zoia MC, Colato $\mathrm{S}$, et al. Long-term durable benefit after whole lung lavage in pulmonary alveolar proteinosis. Eur Respir J. 2004;23(4):526-31.

28. Luisetti M, Rodi G, Perotti C, Campo I, Mariani F, Pozzi E, et al. Plasmapheresis for treatment of pulmonary alveolar proteinosis. Eur Respir J. 2009;33(5):1220-2.

29. Rodríguez Robles AO, Ángel Ortega M, Valenciano López C, Araújo J, Ortiz Barraza E, Pérez Rodríguez. Proteinosis alveolar y lavado broncoalveolar terapéutico(LBA-T). Tratamiento solo o combinado.Patología Respiratoria. 2014;17(2). 